\title{
Regulation of human glioma cell apoptosis and invasion by miR-152-3p through targeting DNMT1 and regulating NF2
}

\section{MiR-152-3p regulate glioma cell apoptosis and invasion}

\author{
Jin Sun ${ }^{1,2}$, Xinhua Tian², Junqing Zhang ${ }^{2}$, Yanlin Huang ${ }^{2}$, Xiaoning Lin ${ }^{2}$, Luyue Chen $^{2}$ and Shizhong Zhang ${ }^{1 *}$ (D)
}

\begin{abstract}
Background: MiRNAs are involved in aberrant DNA methylation through regulation of DNA methyltransferases (DNMTs) in the pathogenesis and progression of glioblastomas (GBM). MiR-152-3p was down-expressed in human malignancies, and served as a tumor suppressor. Neurofibromatosis type 2 (NF2) was significantly decreased in GBM tissues with a high level of methylation. However, the link between miR-152-3p, DNMT1 and methylation of NF2 in GBM is not clearly established. This study was conducted to detect the mechanism between miR-152-3p, DNMT1 and NF2 in GBM.

Methods: The levels of DNMT1 and NF2 expression were studied by qRT-PCR, Western blot, immunofluorescence, and immumohistochemical staining. Methylation in the promoter region of NF2 was detected by methylation-specific PCR and bisulfate genomic sequencing PCR. Cell proliferation was examined by Cell-Counting Kit- 8 and 5-ethynyl-2'-deoxyuridine assay, and cell invasion was evaluated by transwell assay. Flow cytomery and Hoechst staining were used to analyze cell apoptosis. A dual luciferase system was used to confirm the relationship between miR-152-3p and DNMT1.
\end{abstract}

Results: Methylation of NF2 and DNMT1 was markedly increased, and miR-152-3p was downregulated in GBM tissues and glioma cells. Both knockdown of DNMT1 and overexpression miR-152-3p showed that demethylation activated the expression of NF2. Furthermore, miR-152-3p directly targeted DNMT1. Both miR-152-3p overexpression and DNMT1 knockdown significantly induced cell apoptosis and inhibited invasive activity. This was also observed after NF2 overexpression.

Conclusions: These results indicated that miR-152-3p can inhibit glioma cell proliferation and invasion activities by decreasing DNMT1. The restoration of miR-152-3p may have therapeutic application in the treatment of GBM.

Keywords: Glioblastomas, NF2, DNMT1, miR-152-3p, Demethylation

\footnotetext{
*Correspondence: zhangshizhong@smu.edu.cn; zhangshiz168@163.com

'Department of Neurosurgery, Zhujiang Hospital Southern Medical

University, National Key Clinical Specialty, Engineering Technology Research

Center of Education Ministry of China, Guangdong Provincial Key Laboratory

on Brain Function Repair and Regeneration, Guangzhou, Guangdong 510282,

China

Full list of author information is available at the end of the article
} 


\section{Background}

Glioblastoma, also known as glioblastoma multiforme (GBM), is the most common and aggressive type of brain tumour in adults and accounts for about 69\% of all gliomas [1, 2]. GBM is characterized by extensive infiltration throughout the brain parenchyma, robust angiogenesis and necrogenesis, intense resistance to apoptosis, and genomic instability [3]. The current standard conventional treatment for GBM includes neurosurgery, radiotherapy and chemotherapy [4]. However, these aggressive treatments are not effective in controlling the disease, and the prognosis remains dismal, with a median survival of 12-18 months [5]. Therefore, a better understanding of the molecular mechanism of invasiveness and proliferation of GBM cells is important in the development of a more effective therapeutic target in GBM.

The neurofibromatosis type 2 (NF2) gene is a tumour suppressor gene, and its inactivation is important in the development of NF2-associated tumours. The product of the NF2 gene is termed neurofibromin 2 (NF2) protein, also known as merlin or schwannomin. The protein functions as a linker between transmembrane proteins and the actin cytoskeleton and regulates cytoskeleton remodelling [6]. There is growing evidence that demonstrates its critical role in governing cell survival, motility and invasiveness. Recent work on the tumour suppressor function of NF2 has shown that it is dramatically downregulated in malignant gliomas, resulting in enhanced proliferation of glioma cells, and that it plays a pivotal role in tumorigenesis [6-8].

Many factors are involved in the regulation of NF2 activity, such as gene mutation, expression and phosphorylation [9]. It is well recognized that the silencing of tumor suppressors and oncogenes can result from DNA methylation. Disruption of methylation has been observed in GBM. DNA methylation at the 5-position of cytosine is initiated and maintained by catalysis of DNA methyltransferases (DNMTs) [10]. Aberrant DNMT expression has been shown to facilitate tumorigenesis and development [11]. Such epigenetic changes are potentially reversible and therefore are considered promising targets for anti-cancer treatments. Indeed, DNAdemethylating drugs have been approved by the Food and Drug Administration (FDA) as a treatment for myelodysplastic syndromes and myelogenous leukemia $[6,7]$. DNMT1 is a major enzyme responsible for DNA methylation and heavily contributes to the methylation of NF2 in benign meningioma cells and leptomeningeal cells [12]. However, the link between the methylation of NF2 and DNMT1 in GBM is not well understood.

MicroRNAs (miRNAs) are endogenously expressed, short noncoding RNAs of 20-23 nucleotides that target messenger RNA (mRNA). They increase the molecular heterogeneity of GBM and function as micromodulators in the migration and invasion of GBM cells. Therefore, the potential role of miRNAs in the treatment of GBM has become widely recognized. Growing evidence indicates that miRNAs are involved in aberrant DNA methylation through regulation of DNMTs, contributing to tumorigenesis and tumor development. MicroRNA-29 (miR-29) reverts aberrant methylation by targeting DNMT3a and DNMT 3b [13]. MiR-185 is involved in ovarian cancer through targeting DNMT1 [14]. However, the correlation between miRNAs and DNMT1 in GBM, and their role in the development of GBM is largely unknown.

In this study, we investigated the role of miRNAs in the regulation of DNMT1 and NF2 expression, and in the resultant invasiveness of GBM cells. This is the first study to observe that miR-152-3p negatively regulates the expression of DNMT1 and invasiveness of GBM cells. Additionally, the miRNA was found to be involved in the expression of NF2 via methylation. Conclusively, our results suggest a new molecular mechanism underlying regulation of the development of GBM by NF2.

\section{Methods}

\section{Tissue samples from GBM patients}

GBM tissues and adjacent tissues were collected from patients who underwent curative resection at the Xiamen University Affiliated Zhongshan Hospital, Fujian, China. Fresh samples were stored at $-80{ }^{\circ} \mathrm{C}$ in a freezer. The study was approved by the Ethical Committee of Xiamen University Affiliated Zhongshan Hospital, and consent was obtained from all participating patients.

\section{Cell culture and transfection}

The human normal glial cell line, HEB, and the human glioma cell lines U251, U87, T98-G and A172 were obtained from the American Type Culture Collection. The cells are routinely cultured in Dulbecco's modified Eagle media (Hyclone) with 10\% FBS (GIBCO, Life Technologies, Helgerman Court, MD, USA), 100 units/ $\mathrm{ml}$ penicillin and $100 \mathrm{mg} / \mathrm{ml}$ streptomycin in a humidified atmosphere at $5 \% \mathrm{CO}_{2}$ and $37{ }^{\circ} \mathrm{C}$.For transfection, U251 cells were seeded at $5 \times 10^{5}$ cells/well in 6-well plates. The cells were transfected with the DNMT1 siRNA (5' UGUUAAGCUGUCUCUUUCCAAGGAAAGAGAC AGCU UAACAGA $3^{\prime}$ ) or negative control (NC) siRNA with Lipofectamine 2000 (Invitrogen) based on the manufacturer's instruction. Overexpression of miR-152-3p and NF2 were achieved by transfecting the cells with miR152-3p mimics (miR-152-3p) and NF2-overexpressing vector obtained from Genepharma (Shanghai, China) and Vipotion (Guangzhou, China), respectively. The siRNAs, miR-152-3p and their associated control were 
obtained from GenePharma, Shanghai, China. About $48 \mathrm{~h}$ after transfection, the cells were collected for apoptosis determination and expression analysis by qRT-PCR, Western bot and methylation-specific PCE.

\section{Quantitative real-time PCR (qRT-PCR)}

The expression of NF2, DNMT1 mRNA and miR-152$3 p$ was determined with real-time quantification PCR. Total RNA was extracted from U251 cells or tissue samples using the TRIzol reagent (Takara) according to the manufacturer's protocol. Total RNA $(1 \mu \mathrm{g})$ was reverse transcribed with a Bestar ${ }^{\mathrm{rm}} \mathrm{qPCR}$ RT kit (Applied Biosystems) in a $20 \mu \mathrm{l}$ PCR reaction. The levels of the mRNAs and miRNA were quantified by real-time PCR (Stratagene Mx3000P) using DBI Bestar SybrGreen qPCR master Mix. To determine the levels of NF2 and DNMT1 mRNA, RT-PCR was performed with the following primers: NF2, forward: CCCCCAACTCCCCTTTCC ,reverse: AGCCCT TTAGCCCCCCTG and DNMT1, forward: GACCCAC GAAAGCCACC, reverse: CACCTCACAGACGCCACA. The $\beta$-actin gene was used as an internal control. To quantify the level of primary miRNA, qRT-PCR was performed using primary miR-152-3p-specific primers (forward: ACACTCCAGCTGGGTCAGTGCATGACA G, reverse: CTCAACTGGTGTCGTGGAGTCGGCAA TTCAGTTGAGCCAAGTT). The miRNA expression was normalized to the small nuclear RNA U6. All quantitative qRT-PCRs were performed in triplicate using the $2-\Delta \Delta \mathrm{Ct}$ method.

\section{Methylation specific PCR}

Methylation Specific PCR (MSP) was performed with $4 \mu \mathrm{l} / 40 \mathrm{ng}$ bisulfite-modified DNA and $25 \mu \mathrm{l} \mathrm{PCR} \mathrm{mix-}$ ture with $1.8 \times$ PCR buffer, $5 \mathrm{mM} \mathrm{MgCl} 2,100$ pmoldeoxynucleotide triphosphates, primers $(100$ pmol per reaction) and one unit of Taq Platinum (Invitrogen). PCR amplification was conducted at $95{ }^{\circ} \mathrm{C}$ for $3 \mathrm{~min}$, followed by 4 cycles $\left(94{ }^{\circ} \mathrm{C}\right.$ for $1 \mathrm{~min}, 60^{\circ} \mathrm{C}$ for $30 \mathrm{~s}$, and $72{ }^{\circ} \mathrm{C}$ for $45 \mathrm{~s}$ ), and then followed by 28 amplification cycles $\left(94{ }^{\circ} \mathrm{C}\right.$ for $1 \mathrm{~min}, 56{ }^{\circ} \mathrm{C}$ for $1 \mathrm{~min}$, and $72{ }^{\circ} \mathrm{C}$ for $45 \mathrm{~s}$ ). Finally, the elongation was conducted at $72{ }^{\circ} \mathrm{C}$ for $4 \mathrm{~min}$. For parallel quality control, a plasmid containing methylated NF2 sequence and water without DNA template were used as positive and negative control, respectively. The sequences of PCR primers specific for NF2 are given in Table 1. Finally, MSP products were

Table 1 Primers for Methylation Specific PCR of NF2

\begin{tabular}{llc}
\hline Primers & Sequence(5'- 3') $^{\prime}$ & Product Length(bp) \\
\hline NF2-M F & TGCGTTGAAATTAATAATTTACG & 303 \\
NF2-M R & CCAAACTAAAATACAATAACGCGAT & \\
NF2-UF & TGTGTTGAAATTAATAATTTATGA & 303 \\
NF2-UR & CCAAACTAAAATACAATAACACAAT & \\
\hline
\end{tabular}

analyzed using $2 \%$ agarose gel electrophoresis with ethidium bromide.

\section{Bisulfite Sanger sequencing}

A total of $500 \mathrm{ng}$ of genomic DNA extracted from GBM and malignant glioma cell lines was bisulfite converted using a MethylCode ${ }^{\mathrm{Tm}}$ Bisulfite Conversion Kit (Applied Biosystems, USA). The NF2 promoter was amplified by PCR with Taq DNA Polymerase (Invitrogen, USA). The primer sequence was designed using Methyl Primer Express $^{\text {ti }}$ Software v1.0 (Applied Biosystems, USA). Sanger sequencing was performed on the PCR products. Five single molecules were sequenced for each sample.

\section{Western blot assay}

After washing with PBS, the cells were lysed and centrifuged at $14000 \mathrm{~g}$ for $10 \mathrm{~min}$ at $4{ }^{\circ} \mathrm{C}$. The protein concentrations were determined using a BCA protein assay kit. Equal amounts of whole-cell lysates were separated on $10 \%$ SDS-PAGE, then transferred to PVDF membranes (Millipore, Danvers, MA, USA). After blocking with 5\% skimmed milk in TBST at room temperature (RT) for $2 \mathrm{~h}$, the membranes were probed with primary antibodies (anti-NF2 [ab88957] and DNMT1[ab19905], 1:1500 dilution; anti-GAPDH, 1:2000 dilution; Abcam) overnight at $4{ }^{\circ} \mathrm{C}$, and then incubated with the appropriate secondary antibodies. Finally, the probed proteins were visualized using enhanced chemiluminescence solution.

\section{Annexin V assay}

The apoptosis of U251 cells was quantified using a FITC-labeled AnnexinV/propidium iodide (PI) Apoptosis Detection kit (Beyotime, Beijing, China) according to the instructions. Flow cytometric analysis was performed immediately after staining using a flow cytometer (Beckman, USA). Cells in early stage apoptosis werepositive for Annexin V, whereas cells in late stage apoptosis were positive for both AnnexinV and PI positive.

\section{EDU assay and Hoechst staining}

Cell proliferation was detected using a 5-ethynyl-2'-deoxyuridine assay kit (Ribobio, Guangzhou, China; EdU), according to the manufacturer's instructions. Briefly, U251 cells transfected with pcDNA-NF2, DNMT1 siRNA, miR$152-3 p$, or their respective controls were exposed to $50 \mu \mathrm{M}$ EdU for $2 \mathrm{~h}$. After collection, the cells were fixed in $4 \%$ formaldehyde for about $20 \mathrm{~min}$ and then permeabilized with $0.5 \%$ Triton X-100 for about $10 \mathrm{~min}$ at RT. After washing with PBS, the cells were treated with $200 \mu \mathrm{L}$ Apollo ${ }^{\bullet}$ reaction cocktail for $10 \mathrm{~min}$ and then permeabilized with $0.5 \%$ TritonX-100. In addition to detection with flow cytometry, the cells suspended in PBS 
were stained with $100 \mu \mathrm{L}$ Hoechst 33,342 $(5 \mu \mathrm{g} / \mathrm{mL})$ and visualized with a fluorescent microscope (Olympus, Japan).

\section{Luciferase reporter assay}

The luciferase reporter assay was performed with the Dual-Luciferase ${ }^{\circ}$ Reporter (DLR ${ }^{\mathrm{m} x}$ ) Assay System (Promega) according to the manufacturer's instructions. In brief, U251 cells were plated at a density of $2 \times 10^{4}$ cells per well in 24-well plates for $24 \mathrm{~h}$. After incubation, $4 \mu \mathrm{g}$ of plasmids were cotransfected with pMIR-DNMT1-3'UTR-wt/mut and 2 ng of pRL-TK using Lipofectamine 2000 (Invitrogen). The cells were collected after about $48 \mathrm{~h}$ in culture for luciferase assays using the Luciferase Reporter Assay system (GloMax) according to the manufacturer's protocol. The assay was performed in triplicate.

\section{Cell-Counting Kit-8 proliferation assay}

U251 cells, pretreated with pcDNA-NF2, DNMT1 siRNA or the miR-152-3p, were seeded at 2000 cells/well in 96-well plates for $24 \mathrm{~h}$. Cell viability was analyzed using a Cell-Counting Kit-8 (CCK-8) proliferation assay kit following the manufacturer's instruction. Absorption intensity was measured at $450 \mathrm{~nm}$ using a microplate spectrophotometer (TECAN, Australia).

\section{Immunohistochemistry}

Specimens were provided by the Xiamen University Affiliated Zhongshan Hospital, Fujian, China. The study was approved by the Ethical Committee of Xiamen University Affiliated Zhongshan Hospital, and consent was obtained from all participating patients. Surgically resected specimens were fixed in formalin and embedded in paraffin. Expression of NF2 and DNMT1 were evaluated using immunohistochemistry (IHC). After the tissue sections ( $4 \mu \mathrm{m}$ thickness) were deparaffinized and rehydrated, microwave antigen retrieval was conducted in citrate buffer. After cooling, endogenous peroxidase was produced with $3 \%$ hydrogen peroxidase for $5 \mathrm{~min}$ at RT. Then the slides were blocked with $5 \%$ goat serum for $30 \mathrm{~min}$ at $\mathrm{RT}$ and incubated overnight at $4{ }^{\circ} \mathrm{C}$ with primary antibodies against $2 \mu \mathrm{g} / \mathrm{ml} \mathrm{NF} 2$ or $10 \mu \mathrm{g} / \mathrm{ml}$ DNMT1 (Abcam). After being washed in PBS with $0.05 \%$ Tween 20 , the sections were incubated with secondary antibody for $30 \mathrm{~min}$ at RT. After subsequent washes with PBS, DAB was applied for visualization of the indicated proteins.

\section{Immunofluorescence staining}

The treated cells were rinsed with PBS and then fixed with $4 \%$ paraformaldehyde in PBS for $1 \mathrm{~h}$. After rerinsingwith PBS, the cells were permeabilized with $0.1 \%$ Triton X-100 for 2 min on ice, and washed with PBS twice. Immunofluoresence analysis was conducted using specific primary antibodies against rat DNMT1 (Abcam, Cambridge, MA, USA) and against NF2 (Abcam, Cambridge, MA, USA). The cells were subsequently incubated with DAPI detecting liquid for 10s at RT followed by microscopic observation, and results were recorded.

\section{Determination of the invasiveness of GBM cells}

The invasiveness of U251 cells was assessed using Matrigel transwells (BD Biocoat Matrigel Invasion Chamber). U251 cells, pretreated with pcDNA-NF2, DNMT1siRNA or the miR-152-3p, were seeded at $5 \times 10^{5}$ cells/ well in 6-well plates for $12 \mathrm{~h}$. Subsequently, the cells were cultured with serum free medium for $12 \mathrm{~h}$ and then seeded at $2 \times 10^{4}$ cells in $100 \mu \mathrm{l}$ serum-free media per transwell coated with matrigel. In the lower chamber, $700 \mu \mathrm{l}$ complete medium with $10 \%$ FBS was added. After $48 \mathrm{~h}$, the cells were fixed with $4 \%$ paraformaldehyde and stained with crystal violet. Invasiveness was quantified by counting the number of invaded cells from six fields.

\section{Statistical analysis}

Data were presented as mean \pm SD. Statistical differences were analyzed by two-sided Student's t-test. $P$ values less than 0.05 were considered significant.

\section{Results}

NF2 and miR-152-3p were down-regulated, DNMT1 was upregulated in GBM cell lines and tumour tissues

NF2 is widely recognized as an important contributor to GBM. Thus, we investigated its levels of mRNA and protein expression. As shown in Fig. 1a, NF2 mRNA in GBM was significantly decreased compared with normal brain. As DNMT1 plays an important role in the regulation of the expression of various proteins, its expression was evaluated to determine whether it was involved in the downregulation of NF2. As shown in Fig. 1b, we observed that DNMT1 mRNA was significantly increased in GBM compared with matched normal brain control tissue. Because miRNAs are widely accepted as a promising therapy for many diseases, especially tumours, miR-152-3p was investigated by RT-PCR in this study. It was noted that miR-152-3p was significantly decreased in GBM (Fig. 1c). Protein expression levels of NF2 and DNMT1 were determined by Western blot. As shown in Fig. 1d, protein expression of NF2 in GBM was significantly decreased and that of DNMT1 was significantly increased compared with control tissues. These observations were confirmed by IHC detection (Fig. 1f). HEB cells are human normal glial cell line, whereas U251, U87, T98-G and A172 cells are human glioma cells. In these cell lines, we observed that DNMT1 and NF2 were significantly increased and decreased, respectively, in 


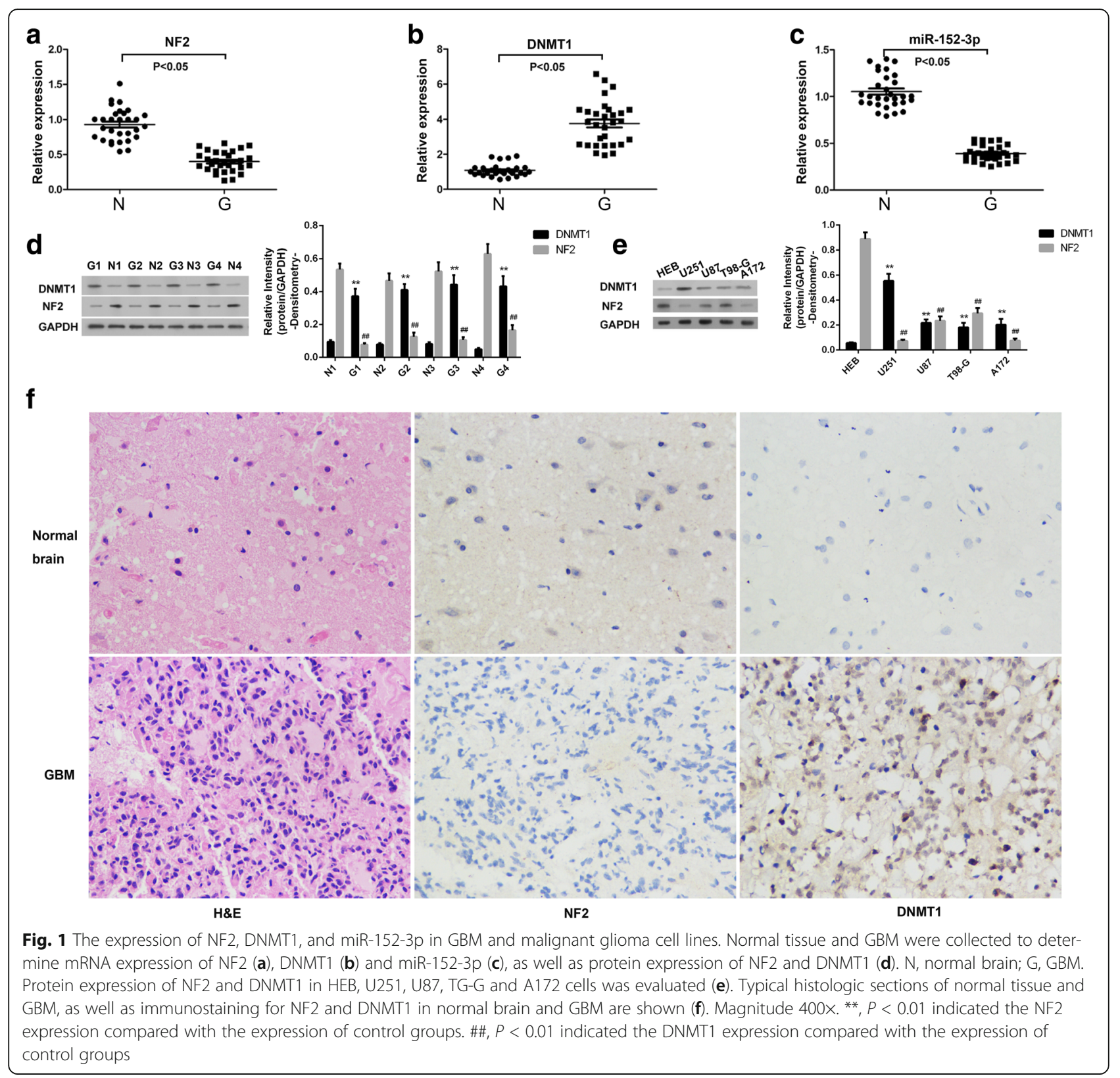

glioma cells compared with HEB cells (Fig. 1e). These data indicated that NF2 might contribute to the development of GBM and that the process might be mediated by DNMT1 and miR-152-3p.

\section{Hypermethylation of NF2}

Given that DNMT1 methylation is critical in DNMT1 regulation of protein expression, the methylation status of NF2 in GBM was assayed in 12 GBM samples versus normal tissue control samples. As shown in Fig. 2a, hypermethylation of NF2 was observed in 10 (83.3\%) of 12 GBM. Unexpectedly, this was also observed in 2 (16.7\%) of 12 normal tissues (data not shown). These results indicated that the incidence of NF2 hypermethylation in GBM was markedly higher than in normal tissues. Based on the high correlations observed between NF2 hypermethylation and GBM, the methylation status of NF2 was potentially involved in GBM. Subsequently, HEB, U251, U87, T98-G and A172 cells were used to determine the methylation status of NF2, to investigate of the correlation between NF2 methylation and GBM. As shown in Fig. 2b, hypermethylation of NF2 was noted in U251, U87, T98-G and A172 cells, but not in HEB cells. In order to further explore whether the methylation sites are located on CpG site sites, which are believed to be associated with decreased gene expression, bisulfite Sanger sequencing was conducted. As shown in Fig. 


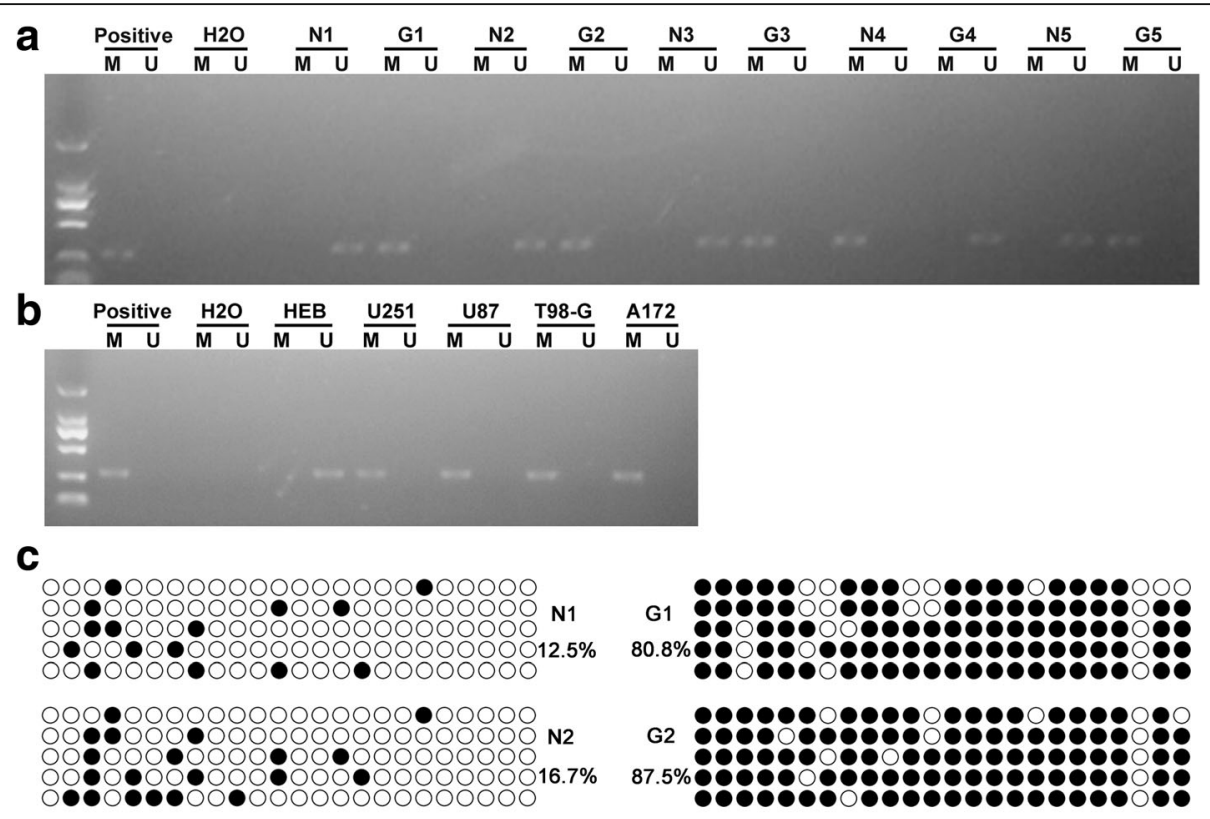

d

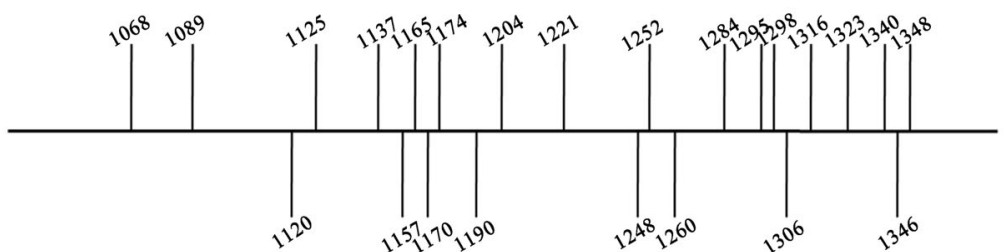

Fig. 2 Hypermethylation of NF2 in GBM and malignant glioma cell lines. The methylation status of NF2 was detected in normal brain and in GBM $(n=5)$. N, normal brain; G, GBM (a). The methylation status of NF2 was detected in HEB, U251, U87, T98-G and A172 cell lines (b). Increased NF2 promoter methylation in GBM brain samples compared with normal brain samples was detected by bisulfite Sanger sequencing (c); the exact methylation sites are indicated $(\mathbf{d})$

2c, NF2 promoter methylation in GBM brain samples was increased compared with normal brain samples, and the exact methylation sites are indicated in Fig. $2 d$. These data suggested that methylation of NF2 might contribute to the development of GBM. This step may be importantly attributed to DNMT1, given the important role of DNMT1 in methylation.

\section{MiR-152-3p directly targeted DNMT1}

MiRNAs regulate the expression of DNMT1 during the pathogenesis of GBM. It has been reported that mir$152-3 p$ is significantly decreased in GBM. Prediction with TargetScan (http://www.targetscan.org/) showed that this miRNA might directly target DNMT1 through a potential binding site in the 3'-UTR of DNMT1 at the position 48-55 (Fig. 3a and b). Two psiCHECK2 luciferase plasmids containing DNMT1-3'UTR and DNMT13'UTR-MUT segments were transfected into HEK293 cells, respectively, together with miR-152-3p to confirm the correlation between mir-152-3p and DNMT1. Luciferase activity was significantly decreased in the wild type (wt)-transfected cells compared with normal controls, and was comparable to the MUT-transfected cells (Fig. 3c). These results indicated that DNMT1 was a direct target of miR-152-3p, the expression of which might affect the biological function of the target.

\section{DNMT1 inhibited the expression of NF2}

In order to confirm whether DNMT1 was involved in the regulation of NF2 expression, loss of function of DNMT1 by siRNA was performed. As shown in Fig. 4a, DNMT1 mRNA expression was significantly decreased after siRNA treatment. As a result, mRNA expression of NF2 and miR-152-3p were significantly increased. Western blot and immunofluorescence showed that, in U251 cells the protein expression of DNMT1 and NF2 were significantly decreased and increased, respectively, after DNMT1 knockdown compared with controls (Fig. 4b and c). Interestingly, DNMT1 was mainly localized to the nucleus, whereas NF2 to the cytoplasm and nucleus. The role of DNMT1 in the hypermethylation of NF2 was investigated in U251 cells. As show in Fig. 4d and e, the NF2 gene was demethylated after DNMT1 knockdown. To further confirm the effect of DNMT1 on the 

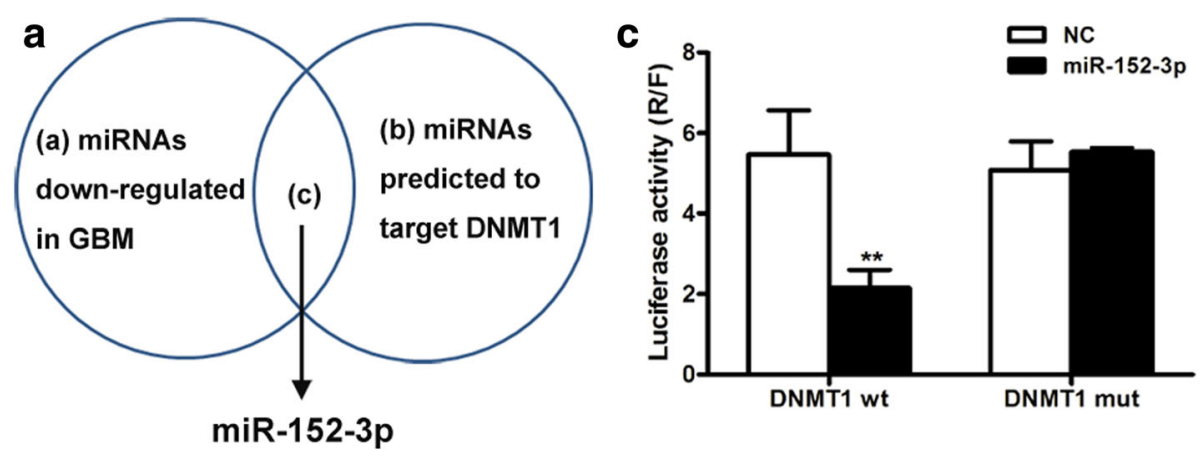

b

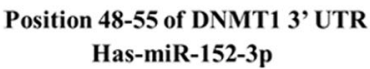

5'...CAGGAAUCCCCCAACAUGCACUGA...
3, GGUUCAAGACAGUACGUGACU

Fig. 3 MiR-152-3p directly targets DNMT1. a MiR-152-3p was predicted to target REST. b Predicted DNMT1 3' -UTR binding sites for miR-12-3p. Alignment of the seed regions of miR-152-3p with DNMT1 3' UTR are shown. c MiR-152-3p directly targets DNMT1. A luciferase reporter vector containing either wild type (wt) or mutated (mut) DNMT1 3'-UTR was co-transfected with miR-152-3p precursor or scramble control in 293 cells, and the luciferase assay was performed. Mean and SEM of 3 independent MSC cultures are shown. ${ }^{*}, P<0.01$ compared with control

NF2 expression, the U251 cells were subjected to the DNMT inhibitor, 5-azacytidine (5-Aza), and the expression of NF2 was detected by RT-PCR and Western blot. Consistent with the results of DNMT siRNA transfection, the expression of NF2 was significantly increased after DNMT inhibitor treatment compared with the PBS-treated group (Additional file 1: Figure S1).

Moreover, in order to further confirm the role of DNMT1 in the regulation of NF2 expression, rescue experiments were performed in which pcDNA-DNMT1 was transfected in the cells. As shown in Fig. 4a and b, DNMT1 mRNA and protein expression levels were significantly increased after pcDNA-DNMT1 transfection, while the expression of NF2 andmiR-152-3p was significantly downregulated. The same pattern was also observed in the immunofluorescence analysis in U251 cells (Fig. 4c). Also, as show in Fig. 4d and e, the NF2 gene was methylated after DNMT1 overexpression. These data indicated that DNMT1 critically contributes, through methylation, to the inhibition of expression of NF2.

\section{MiR-152-3p increased the expression of NF2 through decreased DNMT1 expression}

In order to confirm whether miR-152-3p was involved the expression of NF2, gain of function of miR-152-3p in U251 cells was performed. As shown in Fig. 5a, forced expression of miR-152-3p significantly decreasedDNMT1 mRNA expression. As a result, mRNA expression of NF2 and miR-152-3p was significantly increased. Likewise, protein expression of DNMT1 and NF2 were significantly decreased and increased, respectively, compared with controls (Fig. 5b and c). Furthermore, it was noted that miR-152-3p resulted in the demethylation of NF2 gene (Fig. 5d and e). These data indicated that miR-152-3p inhibited the expression of DNMT1, which led to upregulated expression of NF2 via demethylation.

\section{NF2 overexpression induced cell apoptosis and inhibited cell invasion}

As an important tumour suppressor, NF2 plays a major role in inhibiting the proliferation of cells. As shown in Fig. 6b, Western blot showed that the expression of NF2 was significantly increased when the cell was transfected with pcDNA-NF2. The role of NF2 in the proliferation and apoptosis of cells was then investigated. We observed that NF2 overexpression significantly decreased cell viability at 48 and $72 \mathrm{~h}$ after transfection (Fig. 6a). Flow cytometry (Fig. 6c), Hoechst staining (Fig. 6d), and EDU assay (Fig. 6e) showed that NF2 overexpression significantly induced cell apoptosis and decreased cell proliferation at $48 \mathrm{~h}$ after transfection. The invasive ability of cells critically contributes to tumour development. The effect of NF2 on cell invasion was assessed. As shown in Fig. 6f, the invasion of U251 cells was significantly inhibited by NF2 overexpression compared with control groups, indicating that decreased NF2 might critically contribute to the invasion of cells in GBM.

DNMT1 knockdown and miR-152-3p overexpression induced cell apoptosis and inhibited cell invasion

As an important direct regulator of NF2, the role of DNMT1 in cell viability was assessed. As shown in Fig. 7a, DNMT1 knockdown significantly decreased cell viability at $48 \mathrm{~h}$ after siRNA transfection compared with the control. Similar to the effect of NF2 overexpression, this was confirmed with the apoptosis and proliferation assays (Fig. $7 \mathrm{~b}-\mathrm{d}$ ). Additionally, 

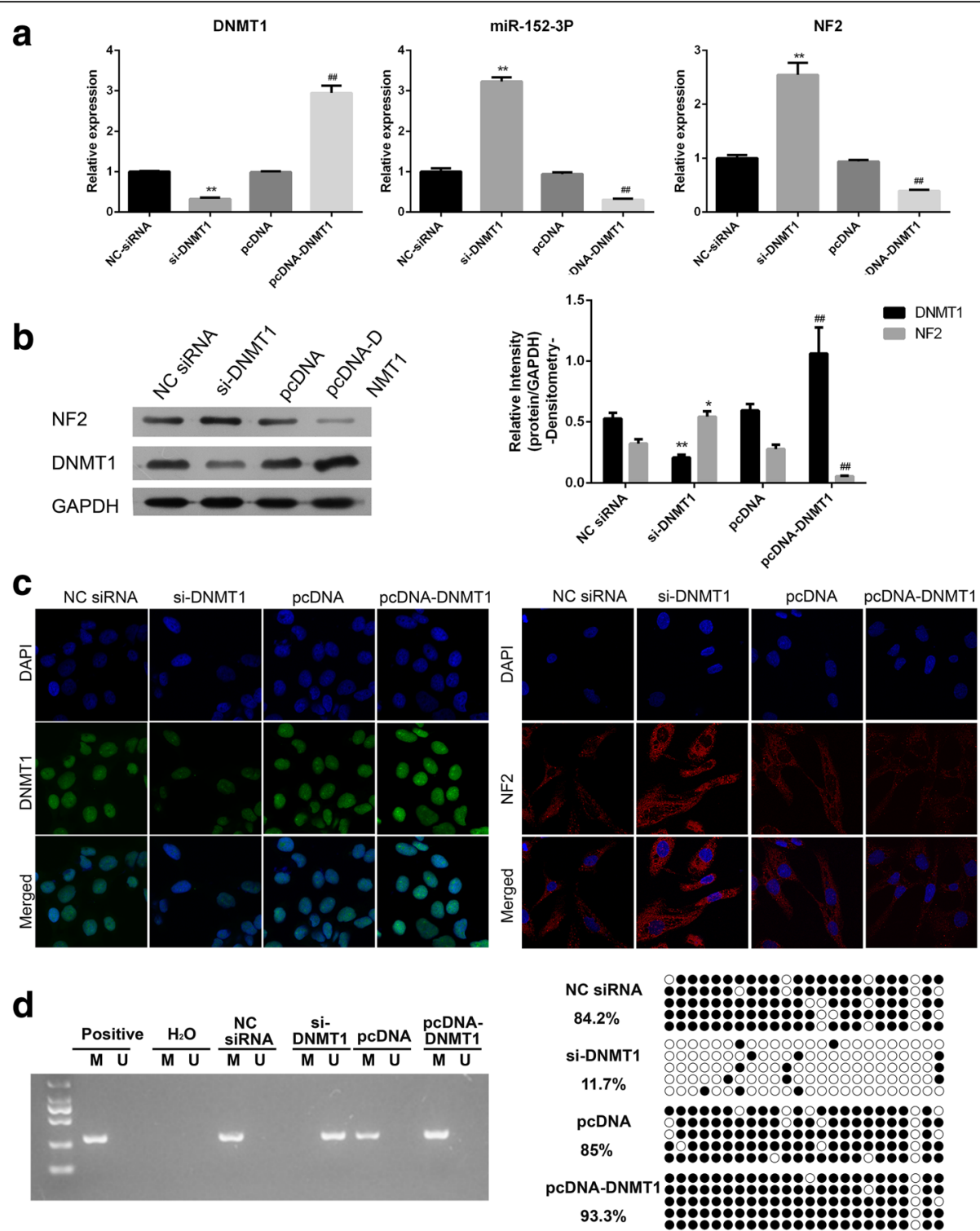

Fig. 4 DNMT1 negatively regulates the expression of NF2. Levels of mRNA expression of DNMT1, NF2, and miR-152-3p after transfection with DNMT1 siRNA and pCDNA-DNMT1 detected by RT-PCR in U251 cells (a). Protein expression of DNMT1 and NF2wasdetected by Western blot (b) and immunofluorescence (c). After DNMT1 knockdown, the methylation status of NF2 in U251 cells was determined with MSP (d) and bisulfite Sanger sequencing (e). ${ }^{*}, P<0.05,{ }^{* *}, P<0.01$ compared with the control groups treated with negative control (NC) siRNA. \#\#, $P<0.01$ compared with control groups treated with pcDNA

DNMT1 knockdown inhibited cell invasion (Fig. 7e). These results indicated that DNMT1 might critically contribute to the cell proliferation and invasion by inhibiting NF2 expression. Subsequently, the effect of miR-152-3p on cell viability was determined. As expected, miR-152-3p overexpression significantly decreased cell viability compared with control treatment (Fig. 8a). Additionally, we observed that miR-152-3p overexpression significantly increased cell apoptosis and decreased the cell invasion compared with the control (Fig. 8b and c).

\section{Discussion}

GBM is the most frequent and aggressive type of glioma. Its high rate of recurrence and resistance to chemotherapeutics predicts a poor prognosis, and highlights the critical need to identify an effective target for therapy. GBM can be induced by different mechanisms, as shown by transcriptional and proteomic. Inactivation and downregulation of the tumour suppressor NF2 has been widely recognized as a critical contributor to GBM. The goal of this study was to explore the underlying mechanism of regulation of the expression 

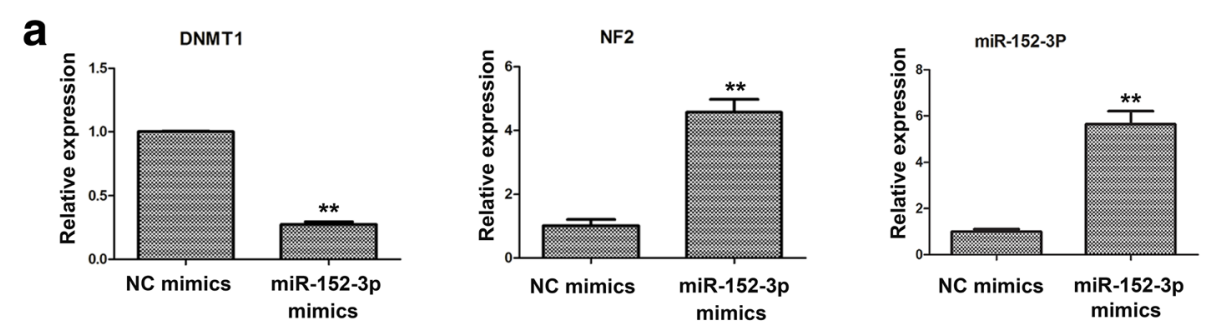

b
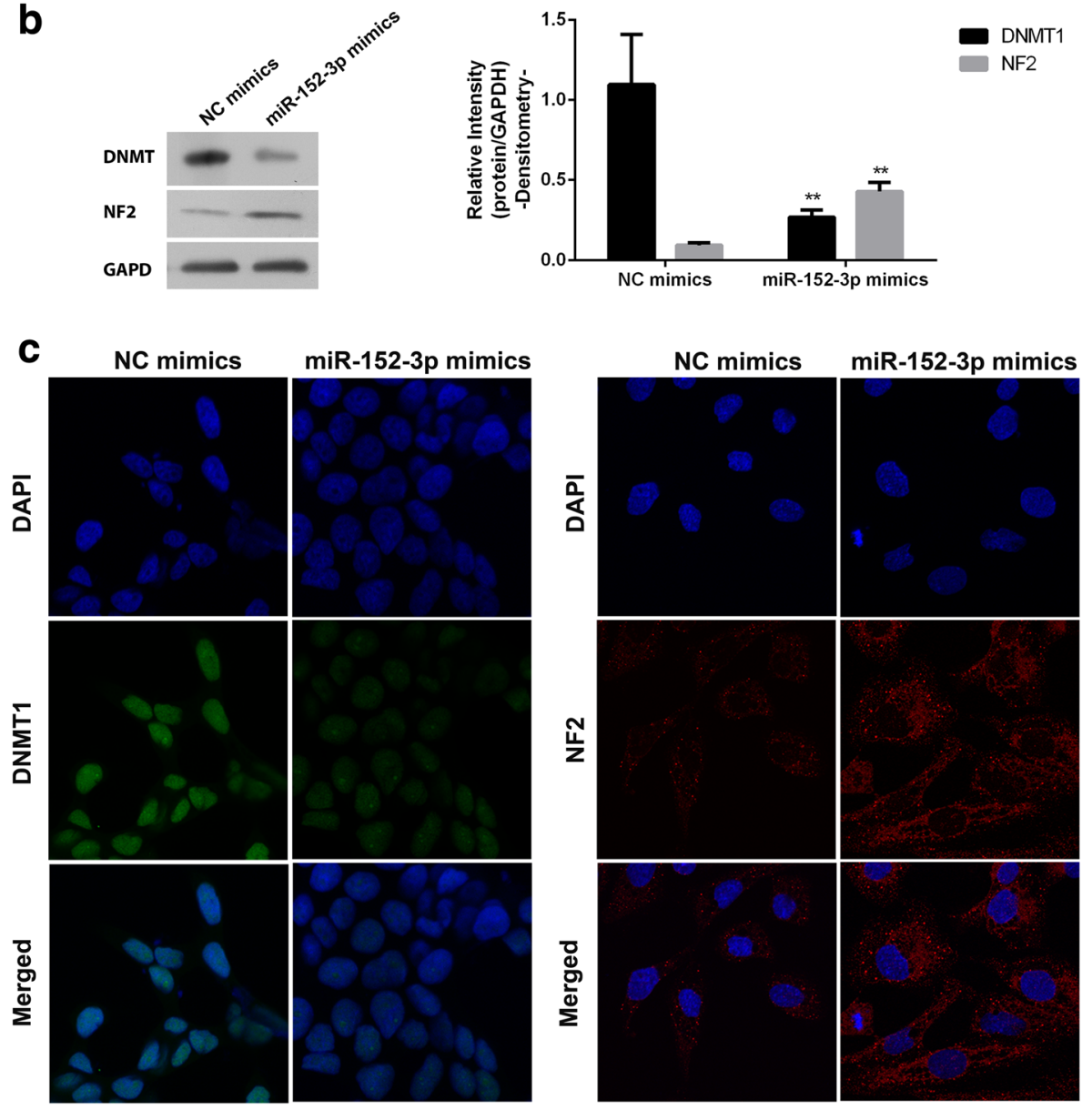

d
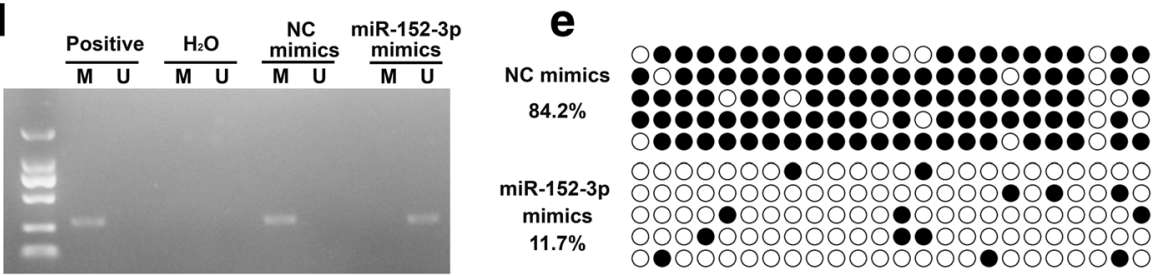

Fig. 5 MiR-152-3p was involved in the regulation of NF2expression.mRNA expression of DNMT1, NF2, and miR-152-3p after transfection with miR152-3p mimics, detected by RT-PCR in U251 cells (a). Protein expression of DNMT1 and NF2 was detected by Western blot after transfection with miR-152-3p mimics (b). The expression of DNMT1 and NF2in U251 cells was detected by immunofluorescence (c). After miR-152-3p transfection, the methylation status of NF2 in U251 cells was determined with MSP (d) and bisulfite Sanger sequencing (e). ${ }^{*}, P<0.01$ compared with control groups treated with NC mimics

of this protein. In this study we showed for the first time that miRNA 152-3p mediates NF2 gene methylation via DNMT1, which critically contributes to the development of GBM. Our results further demonstrate that DNMT1 and miR-152-3p form a negative feedback loop, while NF2 and miR-152-3p form a positive feedback loop. 

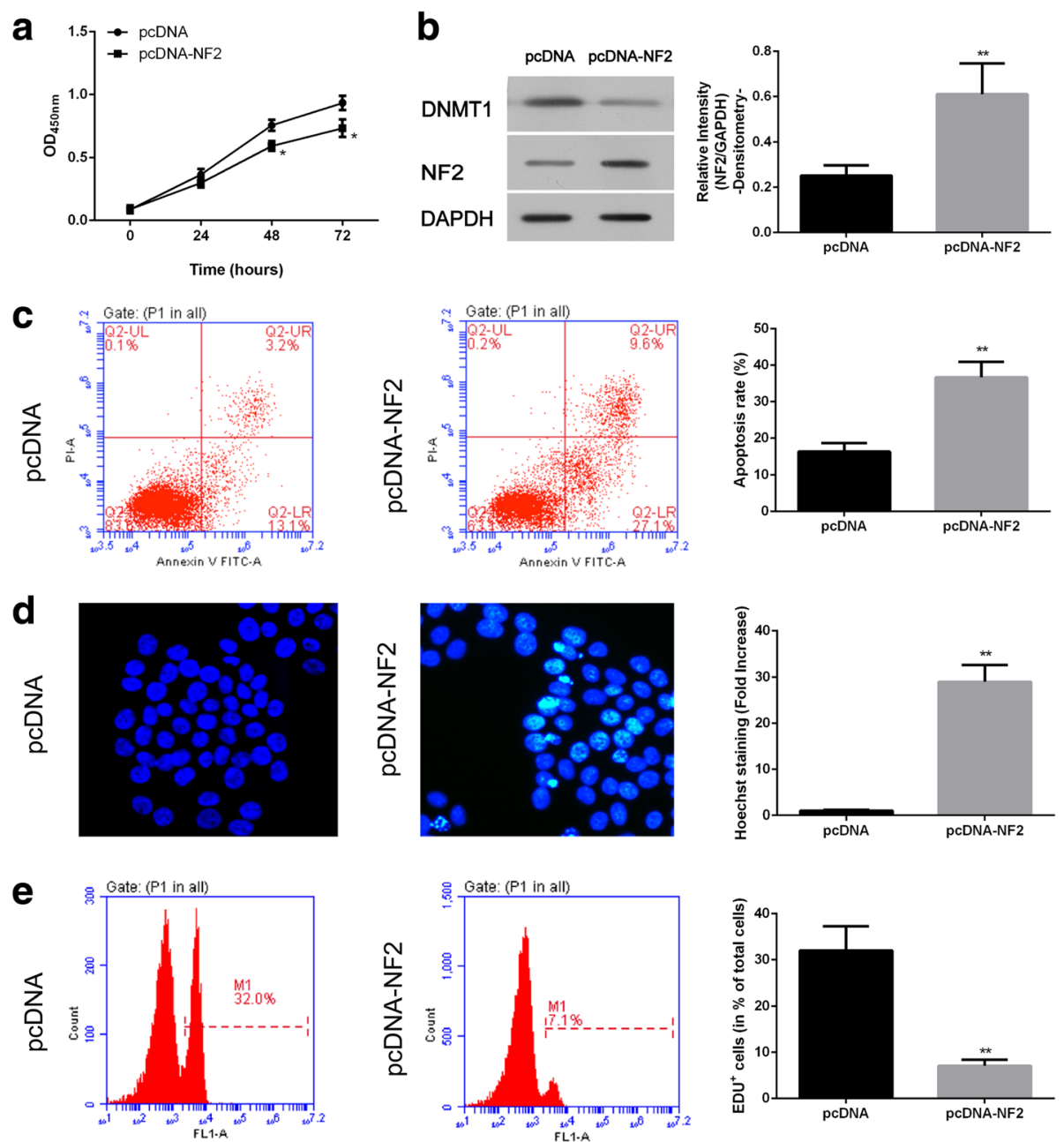

f

pcDNA-NF2
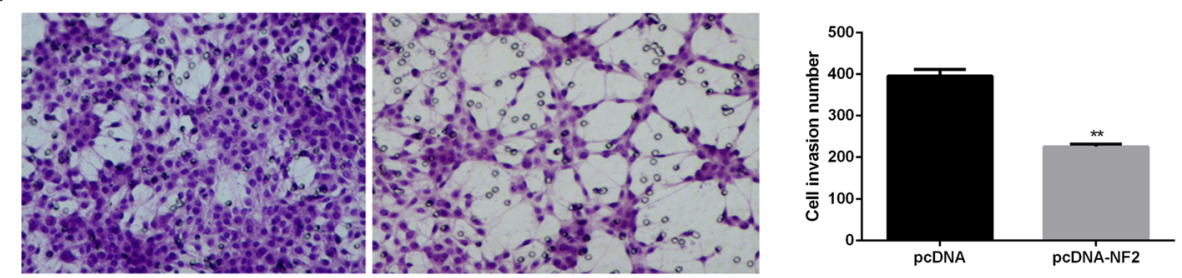

Fig. 6 NF2 was involved in the apoptosis and invasion of U251 cells. The viability of U251 cells was determined at 24, 48 and 72 h after NF2 overexpression (a). Protein expression of DNMT1 and NF2 after transfection with pcDNA-NF2 was detected by Western blot in U251 cells (b). At $48 \mathrm{~h}$ after NF2 overexpression, cell apoptosis was evaluated by flow cytometry (c) as well as Hoechst staining (d), and the cell proliferation was detected with Edu (e); cell invasive activity was detected by transwell (f). ${ }^{*}, P<0.05,{ }^{*}, P<0.01$ compared with control groups

NF2 is a FERM family protein and plays a critical role in the establishment of adherent junctions and is an important mediator of contact inhibition [15]. It is involved in the signalling pathways regulating cell-matrix adhesion, cell proliferation, survival and invasiveness [16]. It has been reported that NF2 activity is regulated by multiple mechanisms. In this study, NF2 was confirmed to be significantly downregulated in the GBM, while DNMT1 was markedly upregulated. Furthermore, we observed hypermethylation of NF2 in GBM tissues, a finding that has not been previously reported. U251, U87, T98-G and A172 are human glioma cells that are routinely used in vitro studies of GBM. Consistent with findings in GBM tumour tissues, NF2 and DNMT1 levels were decreased and increased, respectively, in these cells. Because the contrast was greatest in U251 cells, that cell line was chosen for investigating the correlation between DNMT1 and GBM. 

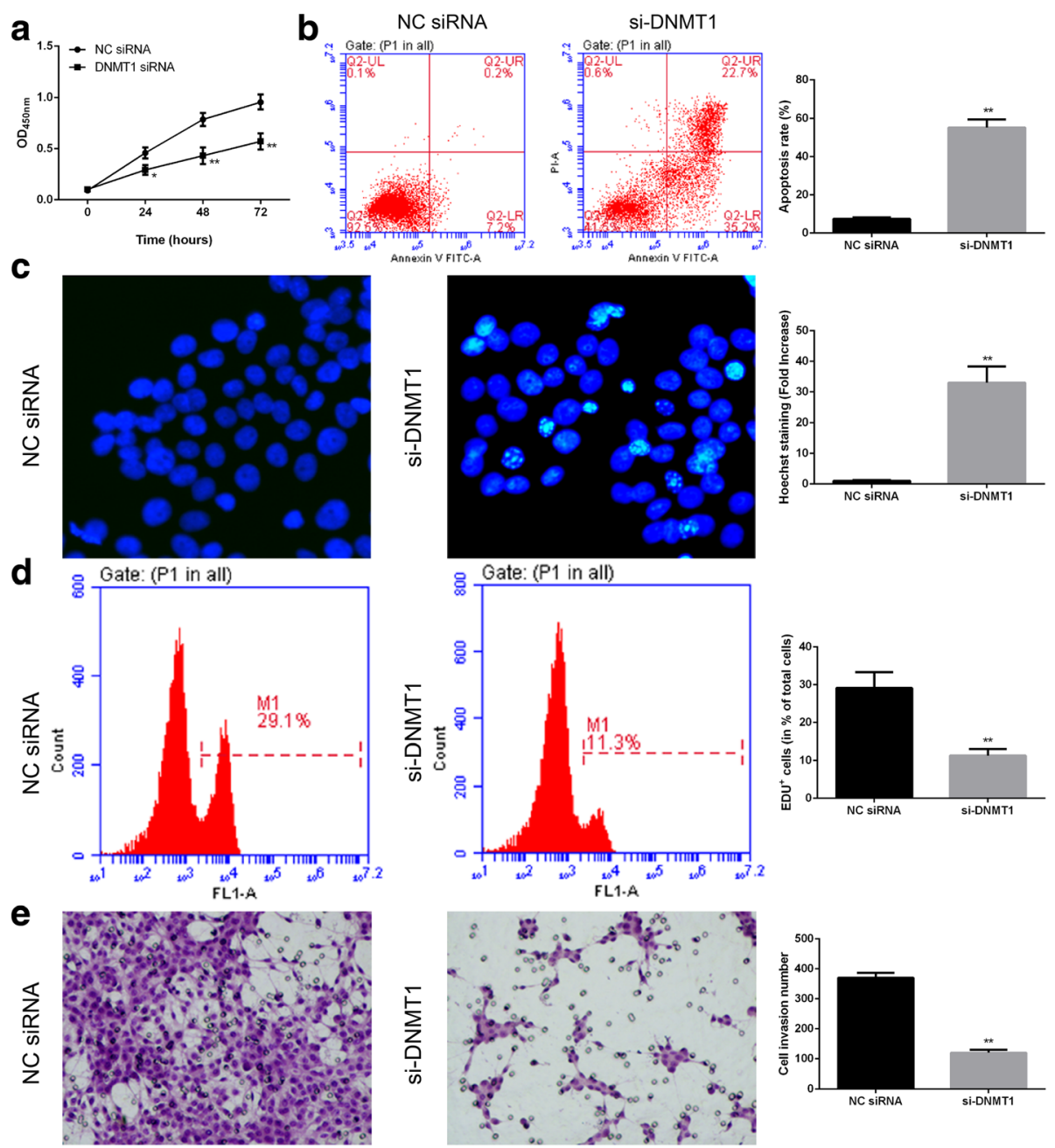

Fig. 7 DNMT1 was involved in apoptosis and invasion of U251 cells. The viability of U251 cells was determined at 24, 48 and 72 h after DNMT1 knockdown (a). At $48 \mathrm{~h}$ after DNMT1 knockdown, cell apoptosis was evaluated by flow cytometry (b) as well as Hoechst staining (c), and cell proliferation was detected with Edu (d); cell invasive activity was detected by transwell $(\mathbf{e}) .{ }^{*}, P<0.05,{ }^{*}, P<0.01$ compared with control groups

Furthermore, because hypermethylation of NF2 was noted in U251 cells, that cell line was also used for further investigation.

The present study showed that in U251 cells, DNMT1 plays a critical role in the proliferation and invasion of GBM cells by decreasing NF2 expression. DNMT1, the maintenance methyltransferase, is widely expressed, particularly in the brain, where there is high expression, and is frequently upregulated in various human cancers such as colon, endometrioid, and prostate [17-19]. Additionally, DNMT1 decreases hTERT expression by hypermethylation, resulting in its reduced expression in glioma cells andenhancedgliomachemosensitivity [20]. ADAMTS9-AS2, a novel tumour suppressor, was modulated by DNMT1 and subsequently contributed to glioma development [21]. Together with the well-recognized role of NF2 in glial cell proliferation in some human malignant gliomas, these findings indicated that increased expression of DNMT1 might promote the development of GBM by decreasing the expression of NF2 through methylation.

It is widely recognized that aberrant miRNAs are involved in various pathogenetic processes [22, 23]. Furthermore, accumulating evidence suggests the presence of different miRNAs with anti-oncogenic properties in glioblastomas [24]. An important mechanism underlying this is their contribution to aberrant DNA hypermethylation through regulation of DNMTs. miR-152 is one of the miRNAs that has attracted considerable interest in recent years, since it is implicated in glioma and other types of cancer $[25,26]$. It targets the 3'-UTR of DNMT1, resulting a significant decrease in DNMT1 at both the mRNA and protein levels. This was confirmed in studies on ovarian cancer, breast cancer, pancreatic cancer and prostate cancer [13, 27-29]. MiR-152-3p, one of the two mature miR-152 sequences, shares the same seed sequence 


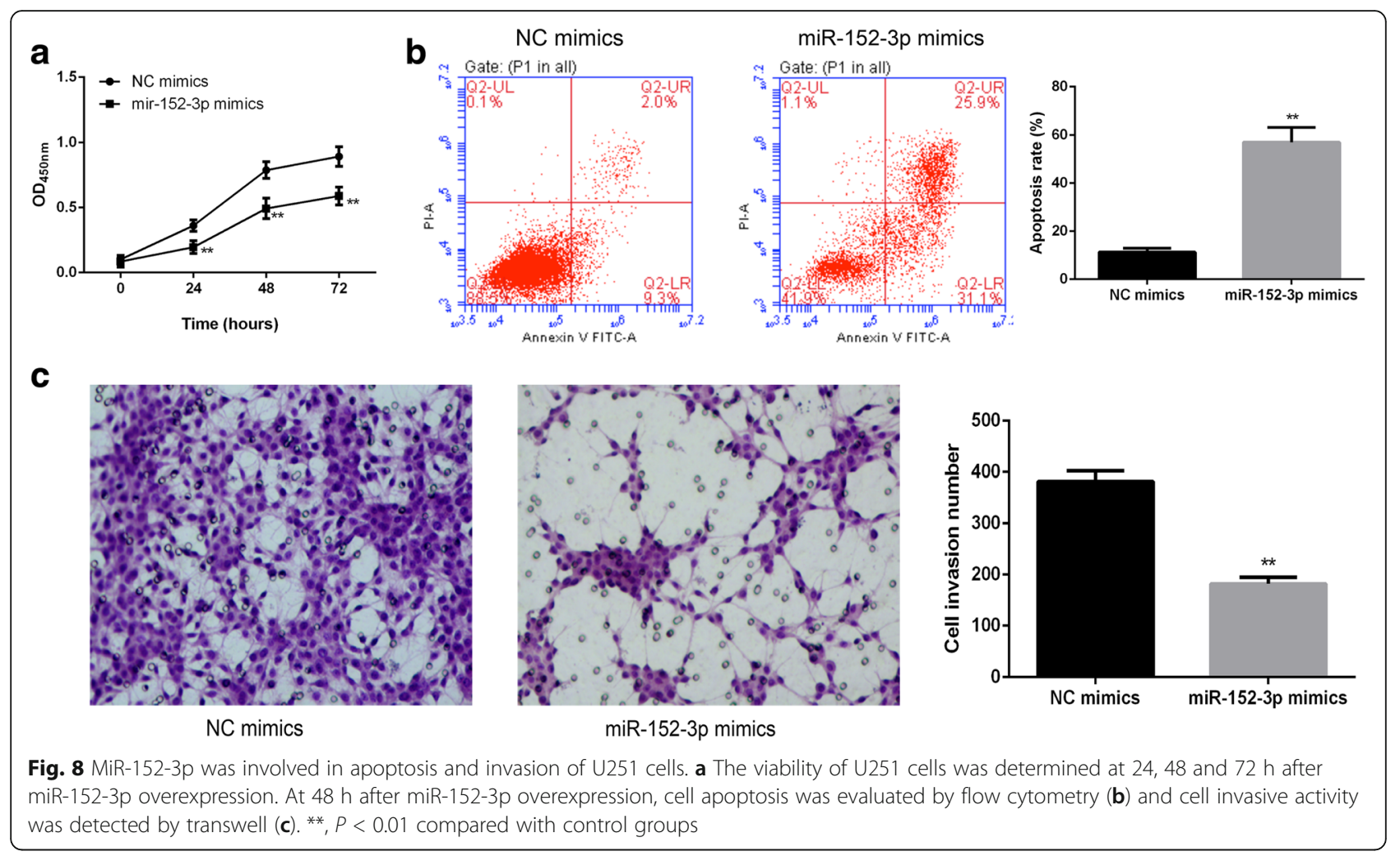

of approximately 6-7 nucleotides with the other members of miR-152 family; the aberrant expression of miR-152-3p was reported to be related to the pathogenesis of tumours such as hepatitis B virus-related hepatocellular carcinoma [13, 30]. In the present study, we showed that miR-152-3p directly targeted DNMT1. We also postulate that overexpression of miR-152-3p significantly enhances demethylation, which further upregulates the expression of NF2. Functionally, miR-152-3p overexpression, DNMT1 knockdown and NF2 overexpression significantly induced glioma cell apoptosis and inhibited their invasion. These data indicated that miR-152-3p might play an important role in GBM suppression via DNMT1-mediated downregulation of NF2.

\section{Conclusion}

In conclusion, our results suggest that miRNA-152-3p functions as a novel regulator to promote glioma cells invasion via DNMT1-mediated downregulation of NF2 and can potentially be used as a treatment for GBM.

\section{Additional file}

Additional file 1: Figure S1. The DNMT inhibitors 5-Azareverses the inhibitory effect of DNMT1 on NF2 expression. Protein and mRNA expression of NF2 in U251 cells after treatment with 5-Aza was detected by RT-PCR (A) and Western blot (B). **, $P<0.01$ compared with the PBS-treated control group. (TIFF $12918 \mathrm{~kb}$ )

\section{Abbreviations}

CCK-8: Cell-Counting Kit-8; DNMTs: DNA methyltransferases; FDA: Food and Drug Administration; GBM: Glioblastoma multiforme;

IHC: Immunohistochemistry; miRNAs: MicroRNAs; mRNA: messenger RNA; MSP: Methylation specific PCR; NC: Negative control; NF2: Neurofibromatosis type 2; PI: Propidium iodide; GRT-PCR: Quantitative real-time PCR

\section{Funding}

This work was funded by National Natural Science Foundation of China (Grant No. 81371397), Xiamen Municipal Science and Technology Bureau Fund Project (Grant No.3502Z20144016) and Fujian Youth Fund Project (Grant No.2015-2-50).

\section{Availability of data and materials}

All data generated or analyzed during this research are included in this manuscript.

\section{Authors' contributions}

JS conceived the study and performed the whole experimental work in its design and coordination. $\mathrm{XT}, \mathrm{JZ}, \mathrm{YH}$ participated in parts of the experiments and analysis of the datas. XL and LC contributed to provide clinical samples. SZ obtained funding and study supervision. The manuscript was written by JS, and revised by SZ. All authors read and approved the final manuscript.

Ethics approval and consent to participate Specimens were provided by the Xiamen University Affiliated Zhongshan Hospital, Fujian, China. The study was approved by the Ethical Committee of Xiamen University Affiliated Zhongshan Hospital, and consent was obtained from all participating patients.

Consent for publication

No applicable.

\section{Competing interests}

The authors declare that they have no competing interests. 


\section{Publisher's Note}

Springer Nature remains neutral with regard to jurisdictional claims in published maps and institutional affiliations.

\section{Author details}

'Department of Neurosurgery, Zhujiang Hospital Southern Medical University, National Key Clinical Specialty, Engineering Technology Research Center of Education Ministry of China, Guangdong Provincial Key Laboratory on Brain Function Repair and Regeneration, Guangzhou, Guangdong 510282, China. ${ }^{2}$ Department of Neurosurgery, Zhongshan Hospital, Xiamen University, Xiamen, Fujian 361004, China.

Received: 1 April 2017 Accepted: 11 July 2017

Published online: 01 August 2017

\section{References}

1. Adamson C, Kanu OO, Mehta Al, et al. Glioblastomamultiforme: a review of where we have been and where we are going. Expert Opinlnvestig Drugs. 2009;18:1061-83.

2. Wang $L$, Bai $Y$, Bao ZS, et al. Hypermethylation of testis derived transcript gene promoter significantly correlates with worse outcomes in glioblastoma patients. Chin Med J. 2013;126:2062-6.

3. Inda MM, Bonavia R, Seoane J. Glioblastomamultiforme: a look inside its heterogeneous nature. Cancers (Basel). 2014:6:226-39.

4. Bastien JI, McNeill KA, Fine HA. Molecular characterizations of glioblastoma, targeted therapy, and clinical results to date. Cancer. 2015:121:502-16.

5. Rankeillor KL, Cairns DA, Loughrey C, et al. Methylation-specific multiplex ligation-dependent probe amplification identifies promoter methylation events associated with survival in glioblastoma. J NeuroOncol. 2014;117:243-51.

6. Lau YK, Murray LB, Houshmandi SS, Xu Y, Gutmann DH, Yu Q. Merlin is a potent inhibitor of glioma growth. Cancer Res. 2008;68:5733-42.

7. McClatchey Al, Saotome I, Mercer K, et al. Mice heterozygous for a mutation at the Nf2 tumor suppressor locus develop a range of highly metastatic tumors. Genes Dev. 1998:12:1121-33.

8. Yi C, Kissil JL. Merlin in organ size control and tumorigenesis: Hippo versus EGFR? Genes Dev. 2010;24:1673-9.

9. Guerrero PA, Yin W, Camacho L, Marchetti D. Oncogenic role of Merlin/NF2 in glioblastoma. Oncogene. 2015;34:2621-30.

10. Subramaniam D, Thombre R, Dhar A, Anant S. DNA methyltransferases: a novel target for prevention and therapy. Front Oncol. 2014:4:80.

11. Wu CT, Lin WY, Chang YH, Lin PY, Chen WC, Chen MF. DNMT1-dependent suppression of microRNA424 regulates tumor progression in human bladder cancer. Oncotarget. 2015;6:24119-31.

12. Wang B, Cui Z, Zhong Z, et al. The role and regulatory mechanism of IL1 beta on the methylation of the NF2 gene in benign meningiomas and leptomeninges. Mol Carcinog. 2016;55:2268-77.

13. Huang J, Wang Y, Guo Y, Sun S. Down-regulated microRNA-152 induces aberrant DNA methylation in hepatitis B virus-related hepatocellular carcinoma by targeting DNA methyltransferase 1. Hepatology. 2010;52:6070 .

14. Xiang $Y$, Ma N, Wang D, et al. MiR-152 and miR-185 co-contribute to ovarian cancer cells cisplatin sensitivity by targeting DNMT1 directly: a novel epigenetic therapy independent of decitabine. Oncogene. 2014;33:378-86.

15. Basu-Roy U, Bayin NS, Rattanakorn K, et al. Sox2 antagonizes the Hippo pathway to maintain stemness in cancer cells. Nat Commun. 2015;6:6411.

16. Ammoun S, Schmid MC, Zhou L, Hilton DA, Barczyk M, Hanemann CO. The p53/mouse double minute 2 homolog complex deregulation in merlindeficient tumours. MolOncol. 2015;9:236-48.

17. Issa JP, Vertino PM, Wu J, et al. Increased cytosine DNA-methyltransferase activity during colon cancer progression. J Natl Cancer Inst. 1993;85:123540.

18. Xiong Y, Dowdy SC, Xue A, et al. Opposite alterations of DNA methyltransferase gene expression in endometrioid and serous endometrial cancers. Gynecol Oncol. 2005;96:601-9.

19. De MAM, Marchi VL, Epstein Jl, Nelson WG. Proliferative inflammatory atrophy of the prostate: implications for prostatic carcinogenesis. Am Pathol. 1999;155:1985-92.

20. Shervington A, Patel R. Silencing DNA methyltransferase (DNMT) enhances gliomachemosensitivity. Oligonucleotides. 2008;18:365-74.
21. Yao J, Zhou B, Zhang J, et al. A new tumor suppressor LnCRNA ADAMTS9AS2 is regulated by DNMT1 and inhibits migration of glioma cells. Tumour Biol. 2014;35:7935-44.

22. Regazzo G, Terrenato I, Spagnuolo M, et al. A restricted signature of serum miRNAs distinguishes glioblastoma from lower grade gliomas. J ExpClin Cancer Res. 2016:35:124.

23. Cheng Z, Wang HZ, Li X, et al. MicroRNA-184 inhibits cell proliferation and invasion, and specifically targets TNFAIP2 in Glioma. J ExpClin Cancer Res. 2015:34:27.

24. Koshkin PA, Chistiakov DA, Nikitin AG, et al. Analysis of expression of microRNAs and genes involved in the control of key signaling mechanisms that support or inhibit development of brain tumors of different grades. Clin Chim Acta. 2014:430:55-62.

25. Zheng X, Chopp M, Lu Y, Buller B, Jiang F. MiR-15b and miR-152 reduce glioma cell invasion and angiogenesis via NRP-2 and MMP-3. Cancer Lett. 2013;329:146-54.

26. Ma J, Yao Y, Wang P, et al. MiR-152 functions as a tumor suppressor in glioblastoma stem cells by targeting Kruppel-like factor 4. Cancer Lett. 2014 355:85-95.

27. Garzon R, Liu S, Fabbri M, et al. MicroRNA-29b induces global DNA hypomethylation and tumor suppressor gene reexpression in acute myeloid leukemia by targeting directly DNMT3A and $3 \mathrm{~B}$ and indirectly DNMT1. Blood. 2009;113:6411-8.

28. Fabbri M, Garzon R, Cimmino A, et al. MicroRNA-29 family reverts aberrant methylation in lung cancer by targeting DNA methyltransferases $3 \mathrm{~A}$ and $3 \mathrm{~B}$. Proc Natl Acad Sci U S A. 2007:104:15805-10.

29. Xu Q, Jiang $Y$, Yin $Y$, et al. A regulatory circuit of miR-148a/152 and DNMT1 in modulating cell transformation and tumor angiogenesis through IGF-IR and IRS1. J Mol Cell Biol. 2013;5:3-13.

30. Wang $S$, Wang $L$, Dou $L$, et al. MicroRNA 152 regulates hepatic glycogenesis by targeting PTEN. FEBS J. 2016;283:1935-46.

\section{Submit your next manuscript to BioMed Central and we will help you at every step:}

- We accept pre-submission inquiries

- Our selector tool helps you to find the most relevant journal

- We provide round the clock customer support

- Convenient online submission

- Thorough peer review

- Inclusion in PubMed and all major indexing services

- Maximum visibility for your research

Submit your manuscript at www.biomedcentral.com/submit
Biomed Central 